FACTA UNIVERSITATIS

Series: Mechanical Engineering Vol. 16, N ${ }^{\circ} 1,2018$, pp. 77 - 86

https://doi.org/10.22190/FUME180102012B

Original scientific paper

\title{
A NUMERICAL STUDY OF THE MICROSCALE PLASTIC STRAIN LOCALIZATION IN FRICTION STIR WELD ZONES
}

\author{
UDC 539.4, 519.6
}

\author{
Ruslan Balokhonov ${ }^{1}$, Varvara Romanova ${ }^{1}$, Ekaterina Batukhtina ${ }^{1}$, \\ Maxim Sergeev $^{2}$, Evgeniya Emelianova ${ }^{2}$ \\ ${ }^{1}$ Institute of Strength Physics and Materials Science, SB RAS, Tomsk, Russia \\ ${ }^{2}$ Tomsk State University, Tomsk, Russia
}

\begin{abstract}
A crystal plasticity approach was used to study the effects of grain shape and texture on the deformation behavior of friction stir weld (FSW) microregions. The explicit stress-strain analysis was performed for two representative grain structures with equiaxed and extended grains. Grain orientations were assigned to simulate no texture or a weak or strong cubic texture. Calculations have shown that the texture gave rise to earlier plastic strain localization on a larger scale. The highest stresses were found to develop in a non-textured specimen with equiaxed grains where the grain boundaries served as a barrier to dislocation motion. In both equiaxed and extended grain structures with a strong cubic texture no pronounced strain localization was seen on the grain scale but mesoscale shear bands appeared early in the deformation process. The calculations have shown that the microstructure-based simulation is a reasonable tool to study the deformation behavior of FSW materials, which is difficult to be predicted within macroscopic models alone.
\end{abstract}

Key Words: Friction Stir Welds, Microstructure, Texture, Crystal Plasticity Simulation

\section{INTRODUCTION}

Friction stir welding (FSW) [1] is an efficient way of joining metals with the use of severe plastic deformation and mechanical mixing of materials in the weld zone at temperatures below the melting point. The solid state of the joined metals is retained during FSW, which is an advantage of this technique over fusion welding. With the FSW

Received January 02, 2018 / Accepted February 07, 2018

Corresponding author: Ruslan Balokhonov

Institute of Strength Physics and Materials Science SB RAS, 634055, Tomsk, Russia

E-mail: rusy@ispms.tsc.ru 
process, it is possible to join materials otherwise difficult or impossible to be welded, specifically aluminum alloys.

A peculiar feature of friction stir welds is a complex microstructure formed in different weld zones which drastically differs from that of the base metal [2-8]. Three distinct regions are commonly observed in FSW joints where the microstructure and mechanical properties gradually vary with a distance from and a depth below the weld surface center line (Fig. 1). The weld nugget (WN) zone is characterized by a fine or ultrafine equiaxed grain structure inherent in dynamic recrystallization [4, 5]. For different materials and FSW regimes, the grain orientations in the $\mathrm{WN}$ region were reported to vary from randomly distributed to those characteristic of a strong shear texture or a cubic texture [4-7]. A thermomechanically affected zone (TMAZ) adjacent to the WN region demonstrates different microstructure patterns on the advancing and retreating sides of the rotated tool [2-8]. A well-defined elongated grain structure is formed on the retreating side. On the advancing side of the TMAZ, columnar-shaped regions of stirringproduced fine grains alternate with the regions of grains inherent from the base metal. Approaching the weld centerline, the grain columns tend to curve about the pin rotation trajectory. For many FSW materials, a two-component texture is shown to form in the TMAZ regions. The heat affected zone (HAZ) has a microstructure similar to that of the base metal, with the mechanical properties being different from those of both base metal and TMAZ due to a redistribution of precipitations $[6,9]$.

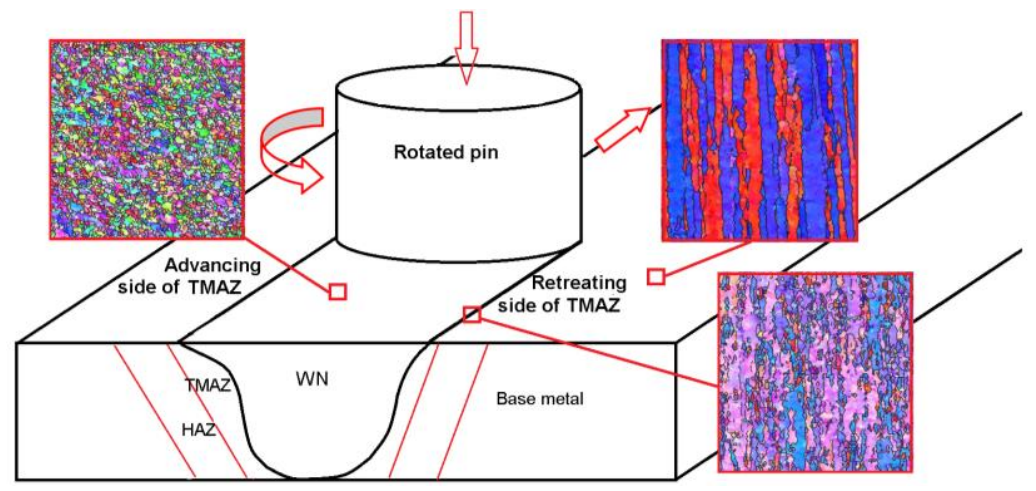

Fig. 1 Schematics of friction stir welding and typical microstructures observed in different FSW regions

Due to a peculiar microstructure, each FSW zone makes a specific contribution to the FSW material deformation and fracture behavior which is difficult to be predicted without consideration of the microstructural effects. Although extensive numerical microstructurebased investigations of micro- and mesoscale deformation phenomena have been performed for many materials, we are aware of only few efforts made to analyze the microscale stress and strain evolution in FSW materials.

In our recent work [9], the finite difference analysis of tensile loading was performed for a microstructure formed in the advancing side of the TMAZ where FSW-affected grains acquired a quasi-rectangular shape and arranged in columns. This type of microstructure was referred to as an ordered microstructure, as distinct from the disordered polycrystalline 
microstructure formed in the nugget and originally seen in the base material. The strength of the material was found to depend on the degree of ordering of the microstructure. A similar numerical analysis for a microstructure containing fine and coarse grains in the nugget and base materials and elongated grains in the TMAZ was performed in [10]. Maximum plastic strain localization and fracture sites were found to depend on the specimen strain and strength of the material. Both sets of calculations $[9,10]$, however, were performed for rather idealized 2D microstructures, with the isotropic elastic-plastic models being used to describe the deformation response of individual grains. It is, therefore, a challenge to perform a more realistic 3D analysis of microscale stress and strain fields formed in different FSW microvolumes under loading with accounting for an anisotropic elastic-plastic material response on the grain scale.

In this paper, we have investigated numerically the grain shape and crystallographic texture effects on the plastic strain localization in microstructures found in the selected FSW zones of an aluminum alloy. Three-dimensional polycrystalline models were constructed to take into an explicit account the grain geometry and the elastic-plastic anisotropy of the facecentered cube (fcc) crystallites.

\section{MicrostruCtURE BASEd SimULATION}

A large group of models implicitly accounting for the grain structure aims at describing a homogenized material response [11,12]. Another approach implies an explicit consideration of the grain structure and thus enables microscale stresses and strains to be estimated [13, 14]. Going this way, we explicitly introduce three-dimensional microstructures found in different FSW zones in finite element calculations. The microstructure-based simulation procedure implies (i) the development of a micromechanical material model accounting for the grain geometry and constitutive behavior, (ii) the model implementation in a boundaryvalue problem complemented with initial and boundary conditions, and (iii) a numerical solution of the problem to study the evolution of stresses, strains, displacements, energy and other parameters of interest under loading.

\subsection{Microstructure model}

Polycrystalline models with equiaxed and extended grains characteristic of the WN and TMAZ, respectively, were generated on regular $3 \times 10^{6}$ element meshes using a method of step-by-step packing (SSP) $[15,16]$. This method is based on a combination of analytical and simulation tools. First, a computational domain is discretized by a mesh, with coordinates being defined for nodal points. Since the design of a microstructure is followed by simulation of its mechanical behavior, the discretization parameters are dictated by the numerical method to be further applied. In a general case, an arbitrarily-shaped computational domain can be discretized by a regular or irregular mesh. As input data, a number of grain seeds are distributed among the mesh elements, with each kind of seeds being associated with a certain analytical law of growth. The laws of seed distribution and growth are derived from experimental data to obtain a model microstructure with geometrical characteristics of the grains similar to real ones. At each further step in the SSP procedure, the volumes surrounding the seeds are incremented by preset values in accordance with the analytical laws of the grain growth. For each mesh element belonging to none of the grains it is checked 
whether coordinates of its central point fall within any of the incremented grain volumes. If so, the cell is considered to belong to this grain and excluded from further analysis. Such a procedure is repeated until the grain structure covers the whole computational domain.

To construct the equiaxed and elongated grain structures presented in Fig. 2, 1600 and 450 grain seeds, respectively, were randomly distributed over a computational domain approximated by a $200 \times 75 \times 200$ regular mesh with cubic elements [16]. In the former case (Fig. 2a), all the grains grew at the same growth rate according to a spherical law, while the growth of extended grains (Fig. 2b) obeyed an ellipsoidal law, with an aspect ratio of the ellipsoidal semi-axes being 1:3.

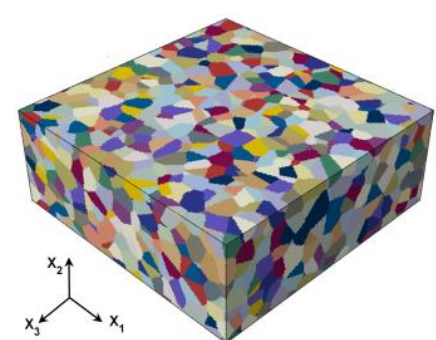

a)

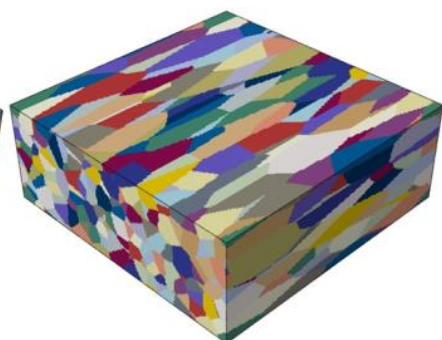

b)

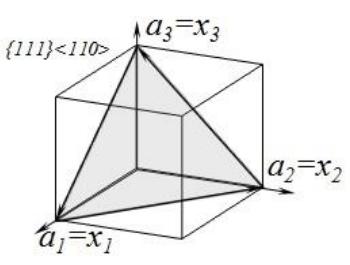

c)

Fig. 2 Polycrystalline models of $200 \times 75 \times 200 \mu \mathrm{m}$ with equiaxed (a) and extended grains (b) and a local coordinate system associated with fcc crystallographic axes

\subsection{Constitutive description of grains}

It is common practice to describe the deformation behavior of a polycrystalline material in terms of the crystal plasticity theory where the polycrystal is treated as an aggregate of single crystals with different crystallographic orientations relative to a global coordinate system [11, 13, 14]. Crystal plasticity models acquire a special significance for materials with a high degree of elastic-plastic anisotropy due to crystallographic texture or a limited number of slip systems [13]. Plastic deformation inside the grains is assumed to occur by dislocation gliding in active slip systems, with the slip system being activated provided that the resolved shear stress becomes equal to a critical value. In a general case, the critical resolved shear stress is a function depending on the strain hardening, microstructure evolution, and etc. A large body of publications devoted to crystal plasticity simulations is available at present (see, e.g., overview [11]). Particularly, a large group of crystal plasticity models were developed for aluminum alloys (e.g., [17]).

The elastic response of grains is described by the generalized Hooke's law for an anisotropic material written in a rate form. Expressing the total strain rate tensor as the sum of the elastic and plastic parts, we get

$$
\dot{\varepsilon}_{i j}=\dot{\varepsilon}_{i j}^{e}+\dot{\varepsilon}_{i j}^{p}
$$

and

$$
\dot{\sigma}_{i j}=C_{i j k l}\left(\dot{\varepsilon}_{k l}-\dot{\varepsilon}_{k l}^{p}\right),
$$

where $\sigma_{i j}$ is the stress tensor and $C_{i j k l}$ is the tensor of elastic moduli. 
Aluminum single crystals have an fcc crystal lattice characterized by a cubic symmetry. Let us write the constitutive equations for an aluminum single crystal with respect to a local coordinate system $x_{i}$ associated with crystallographic axes $a_{i}$ as presented in Fig. 2c. Then, the constitutive equations take on the same form for all grains regardless of their orientations in the global coordinate system $X_{i}$. Due to the symmetry of the crystal lattice, the matrix of elastic moduli in Eq. (2) contains 12 non-zero constants, with only three of them being independent. Components of the plastic strain rate tensor are calculated in terms of the crystal plasticity theory as a summary slip on all active slip systems:

$$
\dot{\varepsilon}_{i j}^{p}=\sum_{\alpha} \dot{\gamma}^{(\alpha)}\left(s_{i} m_{j}+s_{j} m_{i}\right)^{(\alpha)}
$$

where $s_{i}$ and $m_{i}$ are the slip direction and slip plane normal vectors in a slip system $\alpha$. The shear strain rate depends on the ratio between resolved shear stress $\tau^{(\alpha)}$ and threshold stress $\tau_{*}^{(\alpha)}$ in this slip system:

$$
\dot{\gamma}^{(\alpha)}=\dot{\gamma}_{0}\left(\tau^{(\alpha)} / \tau_{*}^{(\alpha)}\right)^{v}, \tau^{(\alpha)}=s_{i}^{(\alpha)} \sigma_{i j} m_{j}^{(\alpha)} .
$$

Here $\dot{\gamma}_{0}$ is the reference slip rate taken to be the same for all slip systems and $v$ is the strain rate sensitivity parameter.

According to Schmid's law, a slip system is activated provided that the resolved shear stress $\tau^{(\alpha)}$ operative on the slip plane $C$ is equal to or greater than threshold value $\tau_{*}^{(\alpha)}$. A large number of models were suggested in the literature to describe critical resolved shear stress $\tau_{*}^{(\alpha)}$ with accounting for different strengthening mechanisms. The more effects are aimed at accounting for, the more fitting and physically-based parameters are required to be determined. Moreover, the iterative methods employed in crystal plasticity calculations additionally increase computational costs. It is reasoned, therefore, to use simplified constitutive models with a reduced number of parameters where possible. In this paper, the following simple expression was used to describe an isotropic hardening of aluminum grains:

$$
\tau_{*}^{(\alpha)}=\tau_{0}^{(\alpha)}+k\left|\gamma_{A}^{(\alpha)}\right|^{b}, \gamma_{A}^{(\alpha)}=\int_{t} \gamma^{(\alpha)} d t
$$

where $\tau_{0}^{(\alpha)}$ is the initial critical resolved shear stress of an aluminum single crystal, assumed to be the same for all slip systems. The second term in this equation defines the strain hardening: $\gamma_{A}^{(\alpha)}$ is the accumulated slip in the slip system $\alpha$ and $k$ and $b$ are the fitting constants.

\subsection{Finite element implementation}

The solution of a boundary-value problem with an explicit account of the microstructure requires for substantial computational resources. On the one hand, the considered microvolume must contain a sufficient number of structural elements (e.g. grains) to simulate micro- and mesoscale deformation processes as realistic as possible. On the other hand, structural elements and interface regions should be approximated in much detail to ensure acceptable accuracy of the solution. This necessitates the use of detailed meshes with a large number of elements. It is, therefore, important for the solution of micromechanical problems to minimize computational requirements without loss of information and solution accuracy. 
An approach that considerably reduces the computer memory, disk space, and computational time requirements suggests the solution of quasi-static problems in a dynamic formulation where equations of motion are solved instead of those of equilibrium in order to find corresponding displacement fields. This allows using explicit numerical methods which have significant advantages over implicit calculations from the viewpoint of computational capacity. The benefit of explicit methods becomes even more critical for solving nonlinear problems such as those concerned with the microstructure-based simulations. In this paper, the deformation behavior of model grain structures was calculated using the finite element software package ABAQUS/Explicit.

The equiaxed and extended grain models were imported in the ABAQUS/Explicit by means of an input file containing an orphan FE mesh, element sets representing grains associated with different local orientations, and the material constitutive parameters appearing in Eqs. (2)-(5). Thus, the grains were associated with the same material but differed by orientations of the local coordinate axes relative to global coordinates, specified in terms of the Euler angles. The material constitutive model, Eqs. (2)-(5), written with respect to the local coordinates, has been imported into the ABAQUS/Explicit by means of a VUMAT user subroutine, with the equations being solved by iterations.

Model parameters and loading conditions were chosen to provide close agreement of quasistatic and dynamic solutions. Shear strain rate $\dot{\gamma}^{(\alpha)}$ being in inverse proportion to the relaxation time was shown to mainly control the material strain rate sensitivity [18]. Calculations for varied loading velocities have shown that the dynamic and static solutions are in a reasonable agreement provided that $\dot{\gamma}^{(\alpha)}$ is approximately ten times as large as the total strain rate. The boundary conditions were formulated with respect to the global coordinate system $X_{i}$ (Fig. 2a). The uniaxial tension was applied along the $X_{1}$-axis in the case of the equiaxed grain structure and along the $X_{1}$ - or $X_{3}$-axis for the extended grain structure. To eliminate dynamic effects, the tension velocity in the both sets of calculations was gradually increased for a time necessary for the elastic wave to run over the computational domain more than 5 times and then kept constant. The top specimen surface was free of external forces and the bottom and lateral surfaces were treated as symmetry planes.

\section{COMPUTATIONAL RESULTS}

\subsection{Stress and strain fields in equiaxed grain structure}

For a polycrystal composed of equiaxed grains (Fig. 2a), three sets of calculations were performed where the grain orientations were distributed at random or scattered about certain crystallographic direction within 5 and $22^{\circ}$ to simulate a strong or weak $\{100\}<100>$ cubic texture, respectively. In all calculations uniaxial tension was applied along the $X_{1}$-axis.

The equivalent stress and plastic strain fields calculated at the initial stage of plastic deformation in the textured and non-textured polycrystals are presented in Fig. 3. The stress distributions in non-textured (Fig. 3d) and weakly textured polycrystals (Fig. 3e) exhibit significant microscopic non-uniformity on the grain scale. In both cases, the highest stress concentration is observed in the vicinity of the grain boundaries. The peak stresses depend on the degree of crystallographic misorientations of contacting grains. This is why a higher level of local stresses is observed in the non-textured material whose grains are characterized 
by a wide range of crystallographic orientations. In the crystal with a strong cubic texture, (Fig. 3f), equivalent stresses demonstrate quasi-uniform distributions. A few stress concentration zones are observed along the grain boundaries, with the stress magnitudes involved being merely twice as high as the average level.

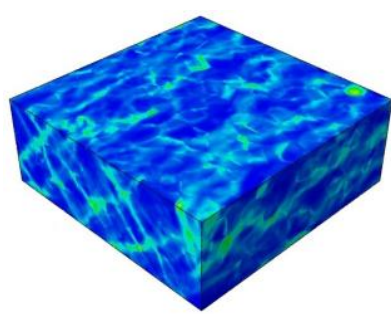

a)

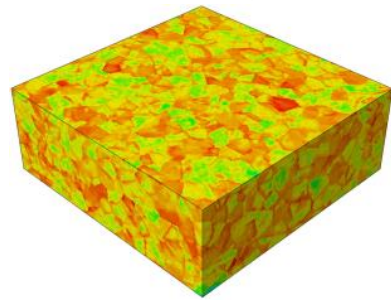

d)

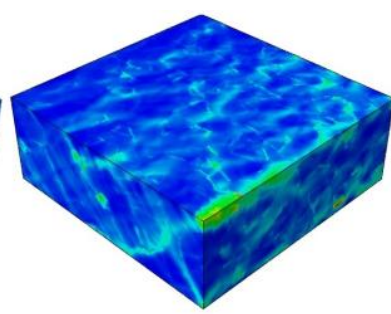

b)

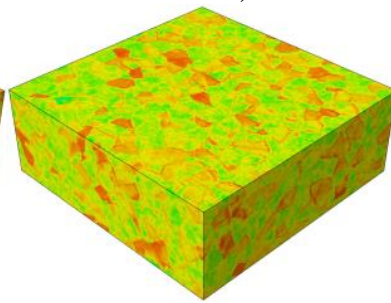

e)

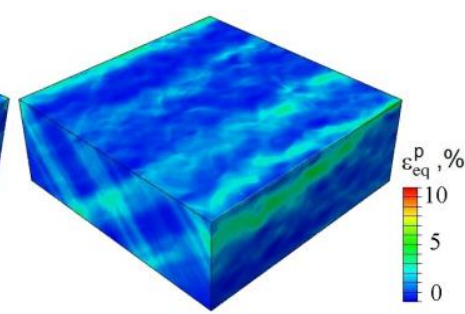

c)

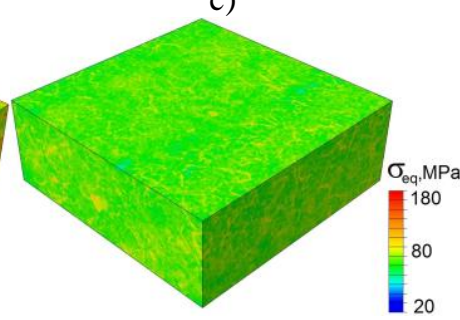

f)

Fig. 3 Equivalent plastic strain $(a-c)$ and stress fields $(d-f)$ in non-textured (a and d), weakly textured (b and e), and strongly textured polycrystals (c and f) at an engineering strain of $1 \%$

The plastic strain distributions demonstrate two characteristic strain localization scales. Smaller-scale strain localization zones are attributed to plastic shear strains developing along the grain boundaries, which gives rise to individual grain displacements and rotations. A distinct strain localization pattern such as this is seen in the non-textured sample (Fig. 3a) and can hardly be found in the strongly textured material (Fig. 3c). Larger-scale strain localization is associated with the formation of shear bands running through the entire sample and involving the whole groups of grains in plastic deformation. The mesoscale bands seen on the top surface are oriented transversely to the tensile axis. On the lateral faces, the bands intersect at an angle of $45^{\circ}$. A well-pronounced mesoscale band pattern is known to be a peculiar feature of many aluminum alloys.

\subsection{Plastic strain localization in extended grain structure}

For a polycrystal consisting of extended grains (Fig. 2b), a strong $\{100\}<100>$ cubic texture was assigned wherein $\langle 100\rangle$ crystallographic direction was chosen to be along the major grain axis. The deviations of the crystallographic axes of grains relative to this direction were specified within $\pm 5^{\circ}$. The polycrystal was subjected to uniaxial tension by two schemes: along and across major grain axis. 


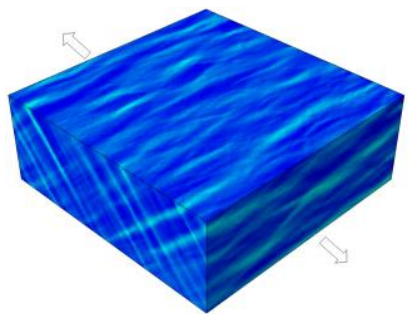

a)

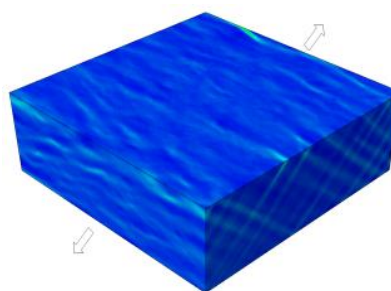

d)

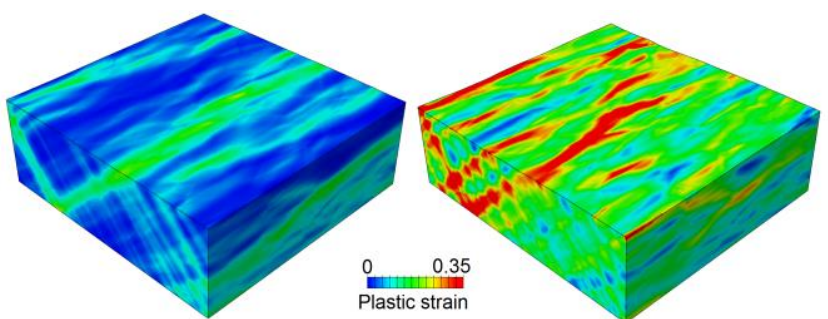

b)

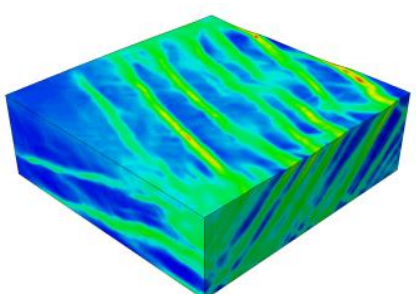

e) c)

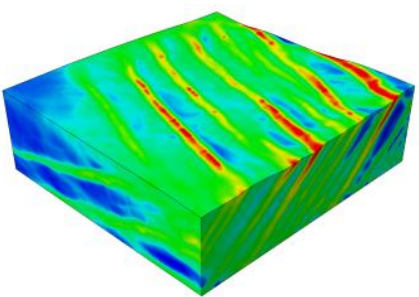

f)

Fig. 4 Equivalent plastic strains in the textured polycrystal composed of extended grains loaded along the $X_{1}-(\mathrm{a}-\mathrm{c})$ and $X_{2}$-axes $(\mathrm{d}-\mathrm{f})$ at an engineering strain of $0.75 \%$ (a and $\mathrm{d}$ ), 3.5\% (b and e) and $7.5 \%$ (c and f)

The plastic strain distributions seen in different tensile stages are presented in Fig. 4. With both of the tensile schemes, the plastic strain distributions bear witness to the strong mesoscale plastic strain localization even on the early plastic flow stage. Notably, the strain localization pattern is hardly affected by the grain boundaries. In other words, the latter are no obstacle to the plastic flow propagation. Without giving illustrations to confirm the foregoing conclusion, it can be emphasized, however, that a stressed state analysis reinforces this statement. The equivalent stress fields exhibit a quasi-uniform pattern, and there are no distinct stress concentration zones even near the grain boundaries. The plastic deformation in the textured material develops at a much lower level of stresses than in a non-textured grain structure.

The localized plastic deformation bands on the free surface are formed across the tensile direction irrespective of grain orientation, whereas on the lateral faces, the bands develop at $45^{\circ}$ to the tensile direction. It should be noted that from the standpoint of the crystallographic texture, directions $X_{1}$ and $X_{3}$ appear to be equivalent. The major difference between the two cases of tension at hand is the relative orientation of the tensile and major grain axes.

\section{CONCLUSION}

Numerical investigations of the plastic strain localization in the microstructures typical of different regions of aluminum friction stir welds. The grain shape and the texture effects were studied using the example of microstructures with equiaxed and extended grains assigned random orientations of crystallographic axes or a weak or strong cubic texture. A crystal plasticity model was applied to describe the elastic-plastic response of aluminum 
grains. The boundary value problem in a dynamic formulation was solved by the explicit finite element method to investigate the deformation behavior of the model microstructures under uniaxial tension.

Calculations have shown that different FSW zones demonstrate distinct deformation response to loading due to peculiar combinations of the grain geometry and crystallographic texture. Both grain shape and texture were found to affect the plastic strain localization patterns to a lesser or greater extent. In all cases, however, the presence of texture gave rise to earlier plastic strain localization on a larger scale. The highest stresses were found to develop in a non-textured specimen with equiaxed grains where the grain boundaries served as a barrier to dislocation motion. On the early deformation stage, the plastic strains in the equiaxed polycrystal mainly localized along grain boundaries, while the larger-scale strain localization in the form of shear bands involving whole groups of grains developed on a later deformation stage. In both equiaxed and extended grain structures with a strong cubic texture no pronounced strain localization was seen on the grain scale but mesoscale shear bands appeared early in the deformation process. Regardless of the grain structure, the mesoscale shear bands run through the whole specimens across the tensile axes, that is, in agreement with experimental evidence for aluminum alloys [19].

In this paper we have considered only few of possible combinations of grain geometry and orientations peculiar for FSW materials with much more effects related to the FSWproduced microstructures, textures and mechanical properties remaining beyond of our study. Nevertheless, we can conclude that the microstructure-based simulation appears to be a reasonable tool to study the deformation behavior of FSW materials, which is difficult to be predicted within macroscopic models alone.

Another important problem disregarded in the paper is concerned with microcrack origination under the FSW processing. The damage problem is of critical significance for FSW applications although in some particular cases the presence of microflaws has negligible effect, e.g., on the adhesive and cohesive strength of internal interfaces [20].

Acknowledgements: This work is supported by the Russian Foundation for Basic Research (grant No. 16-01-00469-a)

\section{REFERENCES}

1. Nandan, R., Debroy, T., Bhadeshia, H.K.D.H., 2008, Recent advances in friction-stir welding - process, weldment structure and properties, Progress in Materials Science, 53, pp. 980-1023.

2. Dumont, M., Steuwer, A., Deschamps, A., Peel, M., Withers, P.J., 2006, Microstructure mapping in friction stir welds of 7449 aluminium alloy using SAXS, Acta Mater. 54, 4793-4801.

3. Mishra, R.S., Ma, Z.Y., 2005, Friction stir welding and processing, Mater. Sci. Eng. R, 50, pp.1-78.

4. K.S.Arora, S.Pandey, M.Schaper, R.Kumar, 2010, Microstructure Evolution during Friction Stir Welding of Aluminum Alloy AA2219, J. Mater. Sci. Tech., 26(8), pp. 747-753.

5. Suhuddin, U.F.H.R., Mironov, S., Sato, Y.S., Kokawa, H., 2010, Grain structure and texture evolution during friction stir welding of thin 6016 aluminum alloy sheets, Mater. Sci. Eng. A, 527, pp.1962-1969.

6. Cho, J., Kim, W.J., Lee, C.G., 2014, Texture and microstructure evolution and mechanical properties during friction stir welding of extruded aluminum billets, Mater. Sci. Eng. A., 597, pp. 314-323.

7. Kumbhar, N.T., Sahoo, S.K., Samajdar, I., Dey, G.K., Bhanumurthy, K., 2011, Microstructure and microtextural studies of friction stir welded aluminium alloy 5052, Mater. Des., 32, pp. 1657-1666.

8. Fonda, R.W., Knipling, K.E., Bingert, J.F., 2007, Microstructural evolution ahead of the tool in aluminum friction stir welds, 58, pp. 343-348. 
9. Balokhonov, R.R., Romanova, V.A., Martynov, S.A., Zinoviev, A.V., Zinovieva, O.S., Batukhtina, E.E., 2016, A computational study of the microstructural effect on the deformation and fracture of friction stir welded aluminum, Comput. Mater. Sci., 116, pp. 2-10.

10. Balokhonov, R.R., Romanova, V.A., Batukhtina, E.E., Martynov, S.A., Zinoviev, A.V., Zinovieva, O.S., 2016, A mesomechanical analysis of the stress-strain localization in friction stir welds of polycrystalline aluminum alloys, Meccanica 51(2), pp. 319-328.

11. Roters, F., Eisenlohr, P., Hantcherli, L., Tjahjanto, D.D., Bieler, T.R., Raabe, D., 2010, Overview of constitutive laws, kinematics, homogenization and multiscale methods in crystal plasticity finiteelement modeling: Theory, experiments, applications, Acta Mater., 58, pp. 1152-1211.

12. Trusov, P.V., Shveykin, A.I., 2013, Multilevel crystal plasticity models of single-and polycrystals, Phys. Mesomech., 16, pp. 99-124.

13. Diard, O., Leclercq, S., Rousselier, G., Cailletaud, G., 2005, Evaluation of finite element based analysis of $3 D$ multicrystalline aggregates plasticity. Application to crystal plasticity model identification and the study of stress and strain fields near grain boundaries, Int. J. Plast., 21, pp. 691-722.

14. Diehl, M., An, D., Shanthraj, P., Zaefferer, S., Raabe, D., 2017, Crystal plasticity study on stress and strain partitioning in a measured 3D dual phase steel microstructure, Phys. Mesomech., 20(3), pp. 311-323.

15. Romanova, V.A., Balokhonov, R.R., Emelyanova, O.S., 2011, On the role of internal interfaces in the development of mesoscale surface roughness in loaded materials, Phys. Mesomech., 14, pp. 159-166.

16. Romanova, V.A., Balokhonov, R.R., Schmauder, S., 2013, Numerical study of mesoscale surface roughening in aluminum polycrystals under tension, Mater. Sci. Eng. A, 564, pp. 255-263.

17. Raabe, D., Sachtleber, M., Zhao, Z., Roters, F., Zaefferer, S., 2001, Micromechanical and macromechanical effects in grain scale polycrystal plasticity experimentation and simulation, Acta Mater., 49, pp. 3433-3441.

18. Romanova, V., Balokhonov, R., Panin, A., Kazachenok, M., Kozelskaya, A., 2017, Micro- and mesomechanical aspects of deformation-induced surface roughening in polycrystalline titanium, Mater. Sci. Eng. A, 697, pp. 248-258.

19. Wittridge, N., Knutsen, R., 1999, A microtexture based analysis of the surface roughening behaviour of an aluminium alloy during tensile deformation, Mater. Sci. Eng. A., 269, pp. 205-216.

20. Popov, V.L., Pohrt, R., Li, Q., 2017, Strength of adhesive contacts: Influence of contact geometry and material gradients, Friction, 5, pp. 308-325. 